\title{
Multilevel Approaches to Reducing Diagnostic and Treatment Delay in Colorectal Cancer
}

\author{
Sherri Sheinfeld Gorin, $\mathrm{PbD}, \mathrm{FSBM}^{1,2}$ \\ ${ }^{1}$ Associate Editor, Annals of Family Medicine \\ ${ }^{2}$ Department of Family Medicine, The University of Michigan School of Medicine, Ann Arbor, Michigan \\ Ann Fam Med 2019;17:386-389. https://doi.org/10.1370/afm.2454.
}

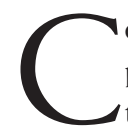

olorectal cancer (CRC) is a major global health problem, with survival varying according to stage at diagnosis. Routine CRC screening identifies precursor lesions at an earlier stage and reduces the risk of death from CRC. ${ }^{1}$ Follow-up after a positive screening test is key, but the probability of undergoing diagnostic colonoscopy after a positive fecal test plateaus at approximately $80 \%$ after 6 months in systems with highly organized screening and at lower levels in systems with less organized follow-up..$^{2,3}$ There is consistent evidence that time to follow-up diagnostic testing after a positive screen is suboptimal, and unequally distributed in many of the European national CRC screening programs, as well as within the United States and Canada. ${ }^{2-10}$ Thus, CRC is still likely to be diagnosed at a late stage after symptoms develop.

The conventional wisdom has been to deliver diagnostic follow-up for a positive screening and subsequent treatment, called the total diagnostic interval (TDI), with minimal delay. In fact, reducing diagnostic and treatment delays has been an axiom of recent national cancer control policies in many western countries, Canada, and Australia, as well as across major health care systems in the United States. As a result, over the past 20 years, policies to reduce the total diagnostic interval have proliferated, particularly at the national level in Europe. The paper by Charles (C. W.) Helsper et $\mathrm{a}^{11}$ in this issue of Annals of Family Medicine contributes to the discussion of diagnostic

Conflicts of interest: author reports none.

\section{CORRESPONDING AUTHOR}

Sherri Sheinfeld Gorin, PhD, FSBM

Department of Family Medicine

The University of Michigan School of Medicine

1150 West Medical Center Drive

Ann Arbor, MI 48104

sherri.gorin@gmail.com and treatment delay among primary care clincians by exploring the reasons for long time to referral for Dutch patients diagnosed with colorectal cancer who presented to general practitioners between 2007-2011. In a retrospective cohort of 309 Dutch patients, the authors analyzed anonymized free text and coded routine primary care data from electronic medical records, linked to the Netherlands Cancer Registry. The paper rests on the Aarhus model ${ }^{12}$ that defines the key time points for diagnostic and treatment pathways, from date of first presentation to diagnosis and treatment, thus enriching the potential generalizability of the findings. For example, the primary care interval is defined as "duration from first consultation with cancer-related signs and/or symptoms in primary care to referral to secondary care." Alongside clinician assessments, well-tested patient and health care system evaluations may contribute to better understanding of diagnostic and treatment delay in this model. And, while questionnaire items from the Aarhus model have been difficult to interpret by patients and clinicians, a more inductive and qualitative approach to collecting these data, as found in this paper, may increase validity of the findings. ${ }^{13}$

Using open coding, axial coding, and selective coding, the authors identified 2 major themes to describe the diagnostic routes to referral of patients with the "longest durations" ( $\geq 90 \%$ of the primary care interval, or $\geq 219$ days): "alternative working diagnosis" and "suboptimal diagnostic strategies"; as well as subthemes of "omitting to reconsider an initial diagnosis" and "lacking follow-up" as reasons for delay. The authors conclude that the long time to referral of CRC in primary care-longer than European benchmarksis primarily related to a low cancer suspicion. Interestingly, given the dominance of the initial hypothesis to medical intervention, they suggest that clinicians consider alternative hypotheses over time. Policy initiatives may increase clinician awareness of the variety 
of CRC symptoms, provide more resources for diagnosis, and offer audit and feedback to providers on compliance with benchmarks for timely referral and follow-up. Technology may assist with the implementation of strict follow-up consultations, as proposed by the authors.

Policy-level interventions to reduce the total diagnostic interval have been implemented across national health care systems in Europe, Australia, Canada, and within large health care systems in the United States. One example of this effort is the International Cancer Benchmarking Partnership, constituted to inform cancer policy in Australia, Canada, Denmark, Norway, Sweden, and the United Kingdom, including reducing delays in diagnosis and treatment. ${ }^{14,15}$ Thus far, guidelines have not been uniformly implemented, but, in 2000, the United Kingdom instituted a twoweek wait rule (TWW) for general practitioners to reduce delay, ${ }^{16}$ in 2015, the Swedish government initiated a national reform to standardize cancer patient pathways and thereby eventually speed up treatment of cancer. ${ }^{17}$ In 2011-2012, Denmark implemented urgent referral pathways to diagnostic centers for nonspecific symptoms, supplementing pathways for alarm symptoms. ${ }^{18}$ Thus far, the results of these policy initiatives on the TDI and cancer mortality have been mixed. The two-week wait is diagnosing a low proportion of patients with CRC overall, although more of these patients are diagnosed with late-stage disease. During the time of the TWW (2000-2006), age-standardized European mortality rates decreased, but these may have been due to the coincident rollout of the national English Bowel Cancer Screening Programme. ${ }^{16}$ The Swedish program has no systematic evaluation yet; in the Danish program, the risk of cancer among patients referred to the urgent referral for nonspecific symptoms was higher than found in previous studies. ${ }^{18,19}$ These programs have not yet been fully implemented across the population.

In the United States, a transdisciplinary team of cancer-specific experts in the Population-Based Research Optimizing Screening Through Personalized Regimens (PROSPR) Consortium has reviewed the literature and developed a consensus statement around the TDI. Using US data in a large organized health care system for $\mathrm{CRC}^{20}$ and modeling estimates, ${ }^{2,21}$ investigators found evidence of an increased risk after delays of 180 days, and a monotonic increase in risk over time. ${ }^{21}$ A consensus recommendation of 90 days for positive CRC screening results is proffered, depending on the resources available and colonoscopy capacity. Based on the available data, wait times longer than 180 days should be avoided. ${ }^{2}$ A 60 -day threshold has been set by the Veterans Health Administration
(VHA) and the Canadian panels. ${ }^{22-24}$ Evaluations of the VHA policy suggest that access to services has been increased and wait time reduced, but their outcomes on morbidity and mortality are not yet known. The Canadian program has had limited evaluation. ${ }^{23,24}$

Within the context of the consensus statement and these policy initiatives, clinicians who have a high index of suspicion of cancer are encouraged to use diagnostic technologies and fast-track pathways for assessment. ${ }^{25}$ Short Message Services (SMS) reminders on cell phones may engage patients and prompt follow-up behaviors. Patient navigation programs have demonstrated effectiveness on CRC screening completion in a recent literature review. ${ }^{26}$ There is some evidence for the cost effectiveness of patient navigation for CRC follow-up, but little is known about the impact of navigation on TDI. ${ }^{26}$

The underlying assumption of these policy, clinician, and patient initiatives is that there is a causal relationship between reduced wait time and reduced mortality. This assumption is not yet consistently supported by the evidence. Because of ethical concerns about withholding treatment from patients with a potential diagnosis of cancer, prospective randomized clinical trials are not possible to test this assumption. Several recent large, prospective, population-based observational studies using newer analytic approaches and adjustments for relevant confounds, seem to suggest the expected relationship across certain time intervals. Tørring et $\mathrm{al}^{27}$ assessed data from general practitioner's questionnaires, interviewer-administered patient questionnaires, and primary care records in 3 population-based studies in Denmark and the United Kingdom. The association between the length of the diagnostic interval and 5-year mortality rate after the diagnosis of CRC was the same for all three types of data, displaying a U-shaped association with decreasing and subsequently increasing mortality with longer diagnostic intervals. The authors conclude that longer diagnostic intervals lead to higher mortality in patients with CRC. Tørring et $\mathrm{al}_{1}{ }^{28}$ reviewing several different cancers collected prospectively from a Danish county registry, found a negative association in length of diagnostic interval and survival in CRC as well as prostate, lung, melanoma skin, and breast cancers. For colorectal cancer in particular they found the same trend when using different sources of information, for different time periods and in two different health care systems (Denmark and the United Kingdom). ${ }^{27}$ They infer from the increasing trends in mortality that a few weeks can make a difference-that time matters.

On the contrary, several systematic reviews of observational studies of CRC, and a recent prospective observational study in Spain with multiple data sources, 
have shown that the diagnostic interval is positively correlated with survival. ${ }^{29-35}$ This relationship is an example of the "waiting time paradox," ${ }^{136}$ in which systematic observation of patients with shorter wait time intervals are diagnosed with more advanced disease and poorer outcomes. Other systematic reviews, ${ }^{37}$ and a study with data from the Danish Cancer in Primary Care cohort, ${ }^{38}$ on a sample of nearly 1,000 patients diagnosed with colorectal cancer in Scotland, ${ }^{39}$ suggest that there is no association between diagnostic and therapeutic delay and survival in colorectal cancer patients.

While definitions of the TDI, measures, and methods differ across these studies, numerous authors have sought to explain the "waiting time paradox" by the presence of certain confounders, such as cancer proliferation rate or tumor aggressiveness. ${ }^{31,32,40}$ Confounding by indication could also influence the findings ${ }^{41}{ }_{i}$ this type of masking occurs when the clinical reason for an intervention or treatment (the predictor) is related to the outcome under study. In this case, clinicians may prioritize follow-up testing for more severe screening findings. Or, diagnostic imaging centers may give priority to more seriously ill patients, and those with "alarm symptoms" such as rectal bleeding, ${ }^{42}$ following them more quickly. These patients may also be at higher risk of more advanced or fatal disease. This type of confounding would distort a positive association between time to diagnostic testing and cancer mortality, such that estimates from analyses are attenuated, null, or even reversed to falsely suggest that a longer wait time is protective. ${ }^{2}$ The presence of patient comorbidities and emergency department admission may also be possible confounders responsible for the waiting time paradox. ${ }^{40,43}$

The data that are collected to assess both TDI and mortality outcomes may also introduce measurement bias. A recent systematic review of psychosocial factors that may impact help-seeking behavior among patients experiencing symptoms found few valid and reliable measurement tools and inconclusive findings across the 35 studies ${ }^{44}$ Additionally, discrepancies in date of diagnosis between patient and clinician recall self-report and electronic medical record data suggest that there may be substantial measurement error in the primary outcome in these studies. ${ }^{29}$ Patient and clinician data may be especially subject to recall bias. There may be differences in registration practices for diagnosis from site-to-site, ${ }^{14}$ and mortality is not always measured as an outcome of policy-level interventions. To address some of these biases, measure selection and future measure development should be guided by psychometric principles. Measures of TDI from more than one source, and by more than one method are optimal, including the systematic application of natural lan- guage processing programs for categorizing narrative data in medical records, and the use of mixed methods to triangulate qualitative and quantitative findings.

Overall, the evidence suggests that the risk for poorer CRC cancer outcomes may rise with longer wait times, which supports performing diagnostic testing as soon as feasible after a positive result. While evidence for specific times for diagnostic testing is limited, benchmarks have been established for varied national and large organized health care systems. Longer diagnostic and treatment wait times may differentially affect disadvantaged groups, leading to increased morbidity and mortality, ${ }_{1}^{10,45,46}$ suggesting possible benefit of targeted interventions for these population subgroups. More research is needed to address the methodological weaknesses in current studies. Reducing diagnostic and treatment delay in CRC is progressing across multiple levels, but there is more work to do.

To read or post commentaries in response to this article, see it online at http://www.AnnFamMed.org/content/17/5/386.

Submitted August 2, 2019; accepted August 5, 2019.

\section{References}

1. US Preventive Services Task Force. https://www.uspreventiveservices taskforce.org.

2. Doubeni CA, Gabler NB, Wheeler CM, et al. Timely follow-up of positive cancer screening results: A systematic review and recommendations from the PROSPR Consortium. CA Cancer J Clin. 2018; 68(3):199-216.

3. Chubak J, Garcia MP, Burnett-Hartman AN, et al; PROSPR consortium. Time to colonoscopy after positive fecal blood test in four US health care systems. Cancer Epidemiol Biomarkers Prev. 2016;25(2): 344-350.

4. Kaplan GS. Health care scheduling and access: a report from the IOM. JAMA. 2015;314(14):1449-1450.

5. Institute of Medicine. Crossing the Quality Chasm: A New Health System for the 21st Century. Washington, DC: The National Academies Press; 2001.

6. Institute of Medicine. Transforming Health Care Scheduling and Access: Getting to Now. Washington, DC: The National Academies Press; 2015.

7. Gingold-Belfer R, Leibovitzh H, Boltin D, et al. The compliance rate for the second diagnostic evaluation after a positive fecal occult blood test: a systematic review and meta-analysis. United European Gastroenterol J. 2019;7(3):424-448.

8. Chen C, Läcke E, Stock C, Hoffmeister M, Brenner H. Colonoscopy and sigmoidoscopy use among older adults in different countries: a systematic review. Prev Med. 2017;103:33-42.

9. Yabroff KR, Washington KS, Leader A, Neilson E, Mandelblatt J. Is the promise of cancer-screening programs being compromised? Quality of follow-up care after abnormal screening results. Med Care Res Rev. 2003;60(3):294-331.

10. Laiyemo AO, Doubeni C, Pinsky PF, et al. Race and colorectal cancer disparities: health-care utilization vs different cancer susceptibilities. J Natl Cancer Inst. 2010;102(8):538-546.

11. van Erp N, Helsper C, Olyhoek S, et al. Potential for reducing time to referral for colorectal cancer patients in primary care. Ann Fam Med. 2019;17(5):419-427. 
12. Weller D, Vedsted P, Rubin G, et al. The Aarhus statement: improving design and reporting of studies on early cancer diagnosis. $\mathrm{Br}$ J Cancer. 2012;106(7):1262-1267.

13. Coxon D, Campbell C, Walter FM, et al. The Aarhus statement on cancer diagnostic research: turning recommendations into new survey instruments. BMC Health Serv Res. 2018;18(1):677.

14. Eden M, Harrison S, Griffin M, et al. Impact of variation in cancer registration practice on observed international cancer survival differences between International Cancer Benchmarking Partnership (ICBP) jurisdictions. Cancer Epidemiol. 2019;58:184-192.

15. Weller D, Vedsted P, Anandan C, et al; ICBP Module 4 Working Group*. An investigation of routes to cancer diagnosis in 10 international jurisdictions, as part of the International Cancer Benchmarking Partnership: survey development and implementation. BMJ Open. 2016;6(7):e009641.

16. Thorne K, Hutchings HA, Elwyn G. The two-week rule for NHS gastrointestinal cancer referrals: a systematic review of diagnostic effectiveness. Open Colorectal Cancer J. 2009;2:27-33.

17. Wilkens J, Thulesius H, Schmidt I, Carlsson C. The 2015 National Cancer Program in Sweden: introducing standardized care pathways in a decentralized system. Health Policy. 2016;120(12):1378-1382.

18. Næser E, Fredberg U, Møller H, Vedsted P. Clinical characteristics and risk of serious disease in patients referred to a diagnostic centre: a cohort study. Cancer Epidemiol. 2017;50(Pt A):158-165.

19. Larsen MB, Hansen RP, Hansen DG, Olesen F, Vedsted P. Secondary care intervals before and after the introduction of urgent referral guidelines for suspected cancer in Denmark: a comparative beforeafter study. BMC Health Serv Res. 2013;13:348.

20. Corley DA, Jensen CD, Quinn VP, et al. Association between time to colonoscopy after a positive fecal test result and risk of colorectal cancer and cancer stage at diagnosis. JAMA. 2017;317(16):1631-1641.

21. Meester RG, Zauber AG, Doubeni CA, et al. Consequences of increasing time to colonoscopy examination after positive result from fecal colorectal cancer screening test. Clin Gastroenterol Hepatol. 2016;14(10):1445-1451, e1448.

22. Peterson K, Carson S, Humphrey L, Helfand M. Patients with Positive Screening Fecal Occult Blood Tests: Evidence Brief on the Relationship Between Time Delay to Colonoscopy and Colorectal Cancer Outcomes. VA-ESP Project 09-199. Portland, OR: Evidence-Based Synthesis Program (ESP), Coordinating Center, Portland VA Medical Center; 2013.

23. Leddin $D$, Bridges RJ, Morgan DG, et al. Survey of access to gastroenterology in Canada: the SAGE wait times program. Can J Gastroenterol. 2010;24(1):20-25.

24. Paterson WG, Depew WT, Paré $P$, et al; Canadian Association of Gastroenterology Wait Time Consensus Group. Canadian consensus on medically acceptable wait times for digestive health care. Can J Gastroenterol. 2006;20(6):411-423.

25. Brown S. Castelli M, Hunter DJ et al. How might healthcare systems influence speed of cancer diagnosis: a narrative review. Soc Sci Med. 2014;116:56-63.

26. Sunny A, Rustveld L. The role of patient navigation on colorectal cancer screening completion and education: a review of the literature. J Cancer Educ. 2018;33(2):251-259.

27. Tørring ML, Frydenberg M, Hamilton W et al. Diagnostic interval and mortality in colorectal cancer: U-shaped association demonstrated for three different datasets. J Clin Epidemiol. 2012; 65: 669-678.

28. Tørring ML, Frydenberg $M$, Hansen $R$ et al. Evidence of increasing mortality with longer diagnostic intervals for five common cancers: a cohort study in primary care. European J Cancer. 2013;49(2): 2187-2198.
29. Leiva A, Esteva M, Llobera J, et al. Time to diagnosis and stage of symptomatic colorectal cancer determined by three different sources of information: a population based retrospective study. Cancer Epidemiol. 2017;47:48-55.

30. Bosch FXJ, Español Llorens J, Campillo Grau M, et al. General analysis of the Tumor Registry of the Hospital de Sta. Caterina de Girona. Description of the series and study of the diagnostic and therapeutic delay, Rev. Española Oncol. 1983;30:209-223.

31. Mulcahy HE, O'Donoghue DP. Duration of colorectal cancer symptoms and survival: the effect of confounding clinical and pathological variables. Eur J Cancer. 1997;33(9):1461-1467.

32. Arbman G, Nilsson E, Störgren-Fordell V, Sjödahl R. A short diagnostic delay is more important for rectal cancer than for colonic cancer. Eur J Surg. 1996;162(11):899-904.

33. Jullumstrø E, Lydersen $\mathrm{S}$, Møller B, et al. Duration of symptoms, stage at diagnosis and relative survival in colon and rectal cancer. Eur J Cancer. 2009;45:2383-2390.

34. García-Peche P, Vázquez-Prado A, Fabra-Ramis R, Trullenque-Peris R. Factors of prognostic value in long-term survival of colorectal cancer patients. Hepatogastroenterology. 1991;38(5):438-443.

35. Terhaar sive Droste JS, Oort FA, van der Hulst RW, et al. Does delay in diagnosing colorectal cancer in symptomatic patients affect tumor stage and survival? A population-based observational study. BMC Cancer. 2010,10:332.

36. Neal RD, Tharmanathan $P$, France $B$, et al. Is increased time to diagnosis and treatment in symptomatic cancer associated with poorer outcomes? Systematic review. Br J Cancer. 2015;112(Suppl 1): S92-S107.

37. Ramos M, Esteva M, Cabeza E, Campillo C, Llobera J, Aguiló A. Relationship of diagnostic and therapeutic delay with survival in colorectal cancer: a review. Eur J Cancer. 2007;43(17):2467-2478.

38. Jensen $H$, Tørring ML, Fenger-Grøn M, Olesen F, Overgaard J, Vedsted P. Tumour stage and implementation of standardised cancer patient pathways: a comparative cohort study. Br J Gen Pract. 2016; 66(647):e434-e443.

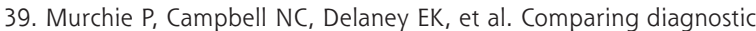
delay in cancer: a cross-sectional study in three European countries with primary care-led health care systems. Fam Pract. 2012;29(1): 69-78.

40. Seoane J, Pita-Fernández S, Gómez I, et al. Proliferative activity and diagnostic delay in oral cancer. Head Neck. 2010;32(10):1377-1384.

41. Tørring ML, Frydenberg M, Hansen RP, et al. Time to diagnosis and mortality in colorectal cancer: a cohort study in primary care. $\mathrm{Br}$ J Cancer. 2011;104:934-940.

42. Koo MM, Hamilton W, Walter FM, Rubin GP, Lyratzopoulos G. Symptom signatures and diagnostic timeliness in cancer patients: a review of current evidence. Neoplasia. 2018;20(2):165-174.

43. Ramos M, Esteva M, Cabeza E, Llobera J, Ruiz A. Lack of association between diagnostic and therapeutic delay and stage of colorectal cancer. Eur J Cancer. 2008;44(4):510-521.

44. Kummer S, Walter FM, Chilcot J, Scott S. Measures of psychosocial factors that may influence help-seeking behaviour in cancer: a systematic review of psychometric properties. J Health Psychol. 2019; 24(1):79-99.

45. Hvidberg L, Wulff CN, Pedersen AF, Vedsted P. Barriers to healthcare seeking, beliefs about cancer and the role of socio-economic position. A Danish population-based study. Prev Med. 2015;71: 107-113.

46. Sheinfeld Gorin S, Heck JE, Cheng B, Smith SJ. Delays in breast cancer diagnosis and treatment by racial/ethnic group. Arch Intern Med. 2006;166(20):2244-2252. 